\title{
Effect of annealing on the size dependent deformation behavior of thin cobalt films on flexible substrates
}

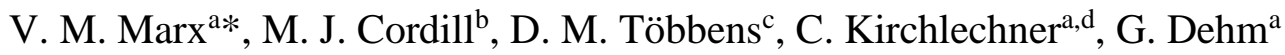 \\ ${ }^{a}$ Max-Planck-Institut für Eisenforschung GmbH, Max-Planck-Str.1, 40237 Düsseldorf, \\ Germany \\ ${ }^{b}$ Erich Schmid Institute of Materials Science, Austrian Academy of Sciences, Jahnstr. 12, \\ 8700 Leoben, Austria \\ ${ }^{c}$ Helmholtz-Zentrum Berlin für Materialien und Energie, Albert-Einstein-Str. 15, 12489 \\ Berlin, Germany \\ ${ }^{d}$ Department of Materials Physics, Montanuniversität Leoben, Jahnstr. 12, 8700 Leoben, \\ Austria
}

*Corresponding author: +49 (0) 2116792 400, marx@ mpie.de, dehm@mpie.de

\begin{abstract}
The effect of film thickness as well as the influence of heat treatment on the deformation behavior of thin cobalt films $(50-2000 \mathrm{~nm})$ on polyimide substrates was investigated using various tensile tests. Straining under an optical light microscope provides information about the fracture strain and cracking behavior. The annealed films exhibit enhanced crack onset strains between 4 and $7 \%$ compared to the as-deposited films with fracture strains of $1-2 \%$. This is partly achieved by a mechanically induced martensitic phase transformation of cobalt from the face-centered cubic (FCC) to the hexagonal-closed packed (HCP) phase. Thereby, it was shown that the heat treatment can be used to increase the amount of metastable FCC phase. Complementary synchrotron diffraction experiments were used to determine the lattice strains which initially increase during straining. After reaching a maximum, the lattice strains decrease in the case of the as-deposited films due to crack formation and in the case of the annealed films due the strain-induced phase transformation and localized plastic deformation in the form of necks. At higher engineering strains, the formation of cracks is also observed in the heat treated
\end{abstract}


samples. Additionally, a decrease of the maximum lattice strain could be found for the HCP phase below a film thickness of $200 \mathrm{~nm}$ and grain size of $50 \mathrm{~nm}$ in the as-deposited films which is caused by cracking.

\section{Introduction}

Cobalt is a common material in magnetic storage devices [1] and can be used in form of cobalt oxide as supercapacitors [2] or as the anode material in lithium ion batteries [3]. Due to the advanced miniaturization of these devices and the consequential geometrical constraints, thin cobalt films exhibit a completely different magnetic and mechanical behavior compared to the bulk material [4-8]. For example, nanocrystalline cobalt shows a two to three times higher yield strength and enhanced ductility at low strain rates compared to polycrystalline cobalt [7]. To further enhance the reliability and lifetime of such devices, it is important to understand the deformation behavior with respect to the film thickness or grain size. A multitude of studies concerning the deformation behavior of thin films with a face-centered cubic (FCC) or bodycentered cubic (BCC) structure like $\mathrm{Cu}, \mathrm{Au}$ or $\mathrm{Cr}$ can be found in literature [9-15]. However, a thorough understanding of the deformation mechanism of hexagonal close-packed (HCP) thin films like cobalt, especially for nanocrystalline cobalt, is still lacking. Only data for Ti films used as an interlayer material are available [16-18].

During cooling cobalt shows a martensitic phase transformation from the FCC to the HCP phase, which is the stable phase at room temperature. In coarse grained cobalt without residual stresses, the transformation temperature is $421^{\circ} \mathrm{C}$ [19]. However, in the presence of metastable FCC cobalt at room temperature, for instance observed in thin films, a mechanically induced phase transformation can occur in the film and could possibly be used to enhance the damage tolerance of such film systems. Thus, the aim of this study is to investigate the mechanical performance of thin cobalt films with film thicknesses between 50 and $2000 \mathrm{~nm}$ in the asdeposited and heat treated state. 


\section{Experimental methods}

The cobalt films were deposited on a $50 \mu \mathrm{m}$ thick polyimide substrate (UPILEX) by sputtering with a pressure of $3.9 \times 10^{-3}$ mbar and $150 \mathrm{~W}$ power. In order to study the influence of the film thickness on the deformation behavior, five different film thicknesses were fabricated: 50, 100, 200, 500 and $2000 \mathrm{~nm}$. Additionally, the effect of annealing $\left(8 \mathrm{~h}\right.$ at $350{ }^{\circ} \mathrm{C}$ in argon atmosphere) was studied so that the samples consist of 50-2000 nm as-deposited and 50-2000 nm annealed cobalt films. Before mechanical testing, phase analysis was performed on all samples in a Seifert X7 X-ray diffractometer with a wavelength of 1,789 Å under grazing incidence $\left(1-2^{\circ}\right)$ from a $2 \theta$ range between 30 and $130^{\circ}$.

In order to study the mechanical behavior, different in situ tensile tests were performed on the as-deposited and annealed cobalt films in a synchrotron and under an optical light microscope (LM). First, the samples cut with a scalpel to a size of $5 \mathrm{~mm}$ x $30 \mathrm{~mm}$ were strained continuously to $12 \%$ engineering strain with an Anton Paar TS600 ${ }^{\circledR}$ straining device in the beam line KMC-2 at BESSY II (Berlin, Germany) [20, 21] and then unloaded to $0 \mathrm{~N}$. Simultaneously, the peaks in the $2 \theta$ range between 46 and $57^{\circ}$ were measured at five $\psi$ angles with a Bruker VÅNTEC area detector. A wavelength of $1.77 \AA$ was selected to avoid X-ray fluorescence from cobalt. The acquisition time of the detector was $10 \mathrm{~s}$ for every frame. From these measurements, the lattice strains in the tensile direction can be calculated with the $\sin ^{2} \psi$ method. Furthermore, the lattice strains allow the determination of the film stresses using the X-ray elastic constant $\left(1 / 2 S_{2}\right)$ for untextured HCP cobalt [22] calculated with the Hill model. The X-ray elastic constants for FCC cobalt at room temperature were not available in literature. In order to provide information about the lattice strains and film stresses of the FCC phase, the (111) FCC and the $(0002)_{\mathrm{HCP}}$ peaks which lie close together are considered separately in the evaluation. The $(111)_{\mathrm{FCC}}$ peak is at a $2 \theta$ angle of $51.25^{\circ}$ and the $(0002)_{\mathrm{HCP}}$ at $51.6^{\circ}$.

Further tensile tests were performed with the Anton Paar TS600 ${ }^{\circledR}$ straining device (asdeposited samples) and with a custom-build screw driven device (annealed samples) under an 
Olympus BX51 optical light microscope and a Zeiss Axio Scope optical light microscope. Therefore, the samples $(1 \mathrm{~cm} \mathrm{x} 3 \mathrm{~cm})$ were strained stepwise until $14 \%$ engineering strain and the images were taken at each straining step. From these LM images, the crack onset strain, i.e. the engineering strain at which the first crack is visible, can be determined.

The averaged crystallite sizes of the as-deposited samples were estimated from the synchrotron data at $0 \%$ engineering strain using the $(10 \overline{1} 0)_{\mathrm{HCP}},(0002)_{\mathrm{HCP}}$ and $(10 \overline{1} 1)_{\mathrm{HCP}}$ peaks and the Scherrer equation $[23,24]$. The instrumental broadening was corrected for using a silicon reference powder. Due to grain growth during annealing, the grain size of the annealed films was determined with electron backscatter diffraction (EBSD) in a Zeiss AURIGA ${ }^{\circledR}$ workstation before straining which provides information of the grain orientation and phase content. The average crystallite and grain sizes of all samples are listed in Table 1.

The post-analysis was performed in the Zeiss AURIGA ${ }^{\circledR}$ workstation which includes a field-emission scanning electron microscope (SEM) and a focused ion beam (FIB) for cutting cross sections in the material to verify if channel cracks are forming which extend across the complete film thickness.

\section{Results}

Before mechanical testing of the cobalt films, qualitative phase analysis was performed in the as-deposited and annealed state of the films. Figure 1a and $\mathrm{b}$ show diffractograms of both film systems with five different film thicknesses. In both sample sets, a mixture of the HCP and FCC phase is present. Due to annealing at $350{ }^{\circ} \mathrm{C}$, changes between the as-deposited and the annealed samples are visible in the diffractogram: the $(10 \overline{1} 0)_{\mathrm{HCP}},(10 \overline{1} 1)_{\mathrm{HCP}}$ and $(11 \overline{2}$ $0)_{\mathrm{HCP}} /(220)_{\mathrm{FCC}}$ peaks decrease while the $(0002)_{\mathrm{HCP}} /(111)_{\mathrm{FCC}},(002)_{\mathrm{FCC}},(01 \overline{1} 2)_{\mathrm{HCP}},(01 \overline{1} 3)_{\mathrm{HCP}}$, $(11 \overline{2} 2)_{\mathrm{HCP}} /(311)_{\mathrm{FCC}},(02 \overline{2} 1)_{\mathrm{HCP}}$ and $(0004)_{\mathrm{HCP}} /(222)_{\mathrm{FCC}}$ peaks increase. Additionally, the annealed peaks are sharper with less background. However, the intensity of the thinnest films is too low for meaningful measurements. The very strong intensity rise of the 
$(0002)_{\mathrm{HCP}} /(111)_{\mathrm{FCC}}$ peak is more pronounced in the synchrotron data (Figure 2). The inserts show that the $(10 \overline{1} 0)_{\mathrm{HCP}}$ and $(10 \overline{1} 1)_{\mathrm{HCP}}$ peaks nearly vanish during annealing.

In order to study the mechanical behavior dependency on the film thickness and heat treatment, different in situ tensile tests were performed. The post mortem SEM images, as illustrated for the 50, 200 and $2000 \mathrm{~nm}$ thick films in Figure 3, show the different cracking behavior of the films. In the case of the as-deposited films, all film thicknesses have relatively straight channel cracks without necking. In contrast, annealed films show a large number of necks forming in between the cracks (see Figure 3). Furthermore, in all systems, the cracks are inclined by $45^{\circ}$ with respect to the film plane independent of the heat treatment as found by analyzing FIB cross sections. This is exemplarily shown for two film thicknesses, $100 \mathrm{~nm}$ and $2000 \mathrm{~nm}$, in both conditions, as-deposited and annealed in Figure 4. This points towards significant plastic deformation rather than brittle failure of the films. It is assumed that the crystals are sheared off which is supported by the fact that adjacent to the "shear crack" a reduction of the grain size is observed. However, neither the phase of the small grains in the strained state nor their parent phases could be determined.

Figure 5 shows the crack onset strains of the as-deposited and annealed films which were determined from the in situ tensile tests performed under the optical light microscopes. It can be observed, that the fracture strain of the as-deposited films is between 1 and $2 \%(+/-0.5$ $\%$ standard deviation) and is much smaller than of the annealed films which have crack onset strains between 4 and $7 \%$ (+/- $1 \%$ standard deviation).

To link the cracking behavior with the lattice strains of the cobalt films, tensile tests were performed at KMC-2 using the $\sin ^{2} \psi$ method. Figure 6 shows the lattice strains and film stresses of selected diffraction peaks (see Figure 2) versus the engineering strain during tensile testing, exemplarily for the as-deposited and annealed $2000 \mathrm{~nm}$ thick film. The lattice strains of the $(10 \overline{10})_{\mathrm{HCP}}$ peak are drawn in black, of the $(111)_{\mathrm{FCC}}$ peak in red, of the $(0002)_{\mathrm{HCP}}$ peak in blue and of the $(10 \overline{1} 1)_{\mathrm{HCP}}$ peak in green with all curves following the same trend. During tensile 
testing, the lattice strains increase reaching a maximum at approximately $1.5-2.5 \%$ engineering strain. After this, the lattice strains decrease until around 7-8 \% engineering strain where a plateau is formed. Independent of the heat treatment, the lattice strains of the (111) FCC peak are in the same range. The values for the $(10 \overline{1} 0)_{\mathrm{HCP}}$ and $(0002)_{\mathrm{HCP}}$ peak are slightly higher for the as-deposited than for the annealed films.

The lattice strains of the 50-200 nm annealed films could not be evaluated due to a strong texture of $\langle 0001\rangle_{\mathrm{HCP}}$ and $\langle 211\rangle_{\mathrm{FCC}}$ with insufficient accessible $\psi$ angles. Furthermore, the intensity of the 50 and $100 \mathrm{~nm}$ thick films was not sufficient for any in situ evaluation. Figure 7 shows the $(10 \overline{1} 1)_{\mathrm{HCP}}$ lattice strains and film stresses for different film thicknesses of the as-deposited and annealed films which were evaluated. In the case of the as-deposited films, no clear trend of the lattice strains with film thickness was observed. Up to an engineering strain of approximately $2 \%$, the lattice strains of the 200 and $500 \mathrm{~nm}$ thick as-deposited films increase revealing a tendency for higher lattice strains than the $2000 \mathrm{~nm}$ thick film. The lattice strain peaks for the annealed films are reached at engineering strains of approximately $1 \%$ followed by a monotonic decrease in lattice strains with increasing engineering strain.

\section{Discussion}

In this study, all investigated films, as-deposited and annealed, consist of the stable HCP and the metastable FCC phase in the unstrained state at room temperature. As shown in a previous work [25], the amount of the FCC phase is enormously increased by the heat treatment, however, decreases during straining due to a strain-induced phase transformation from $51 \%$ to $8 \%$ in case of the $2000 \mathrm{~nm}$ annealed film as measured by EBSD. Thus, besides plastic deformation, grain growth and fracture of the films, the strain-induced phase transformation has to be taken into account. Furthermore, it has to be mentioned that annealing also leads to a change in the microstructure, especially grain size, and most likely the grain boundary structure as well. A separation of the aforementioned mechanisms is not feasible with the applied in situ 
methods and is beyond the scope of this work. However, several scientifically and industrially interesting observations could be made:

(i) Annealing leads to a strong texture formation with sharper X-ray peaks.

(ii) The fracture strain deduced from straining experiments under an optical light microscope is significantly higher for annealed films.

(iii) The evolution of lattice strains during tensile straining of the as-deposited films weakly indicates an expected film thickness or grain size dependency. The maximum lattice strains are smaller for the largest film thickness and grain sizes while the 200 and $500 \mathrm{~nm}$ thick films show similar results for the peak lattice strain within the error bars.

Annealing of the films results in (i) a strong fiber texture with a $\langle 0001\rangle_{\mathrm{HCP}}$ and $\langle 211\rangle_{\mathrm{FCC}}$ fiber axis. The origin of this behavior had not been investigated in this work, but a texture formation caused by heat treatment is well known [26, 27]. Additionally, the peak width after annealing has significantly decreased due to grain growth as well as the annihilation of grownin defects.

The substantially delayed onset of cracking in the annealed films compared to the asdeposited films (ii) is of great interest from an applications viewpoint. It could be shown in a previous work on a $2 \mu \mathrm{m}$ thick annealed cobalt film on polyimide [25], that annealing increases the fraction of the metastable FCC phase and that the strain-induced phase transformation, which begins at $2 \%$ engineering strain, is partially accommodating the applied strains and prevents strain localization and cracking. Therefore, the transformation enhances the ductility leading to a shift of the crack formation to higher fracture strains of 6-7 \% [25]. This leads to the conclusion, that annealing of the cobalt films can enhance the lifetime of devices at application strains below $4 \%$ engineering strain. However, its magnitude is more pronounced for the thinnest and the thickest films as shown in Figure 5. 
All cracks independent of the film thickness and heat treatment are inclined by $45^{\circ}$ through the film thickness and additionally show torn crack flanks. This behavior indicates a significant amount of plastic deformation for the annealed as well as as-deposited films, which was unexpected by the rather brittle-like behavior of the as-deposited films and especially for the thinner films (see Figure 3). It is therefore concluded that the zones are entirely sheared off leading to crack-like deformation patterns. This view is further supported by the small grains in the vicinity of the crack which might be formed during localized shear (as shown in Figure 4 for the $2000 \mathrm{~nm}$ thick annealed film). However, neither the orientation nor the phase in the vicinity of this regions is known prior to straining. Thus, no satisfying conclusion on the failure mechanism can be drawn based on these experiments. Future experiments based on EBSD or $\mu$ Laue diffraction [28] have to be performed to unravel the sequence of damage in these zones.

The lattice strains (iii) of the as-deposited and annealed film follow similar trends which was also observed for the corresponding film stresses on different film systems $[9,15,29,30]$. After reaching a maximum at low engineering strains, a decrease of the lattice strains with increasing engineering strain can be observed until a plateau is formed for the as-deposited films. This plateau in the lattice strains can be correlated to the crack saturation regime. Although showing a similar behavior, the reason for the decrease of the lattice strains at engineering strains above 1-2\% engineering strain is different in both film systems. In case of the as-deposited films, the lattice strains decrease rapidly due to crack formation that begins at 1-2\% engineering strain as determined in Figure 5. In case of the annealed films, as shown in [25], the phase transformation from a metastable FCC to HCP of a $2000 \mathrm{~nm}$ cobalt film starts at $2 \%$ engineering strain. This delays the onset of cracking which is first observed in situ in film systems, that undergo a phase transformation, between 4 and $7 \%$ engineering strain (see Figure 5) and leads to a more uniform decrease of the lattice strains between 2 and 4-7 \% engineering strain (see Figure $6 \mathrm{~b}$ and $7 \mathrm{~b}$ ). Due to the fact that with increasing film thickness the plastic deformation increases, the amount of localized plastic deformation, or necks, also 
increases leading to a reduction of the lattice strains as shown in [25] and Figure 7b. Only at higher engineering strains cracks are also formed further decreasing the lattice strains.

Considering the different film thicknesses, the lattice strains of the as-deposited films reveal only a weak "smaller is stronger" trend previously observed $[9,10,15,29,31]$. This behavior can be explained by the large amount of cracks forming in the thinner films leading to a large reduction of the lattice strains as for instance already described in $\mathrm{Cu} / \mathrm{Cr}$ films [29].

\section{Conclusion}

The film thickness as well as the influence of heat treatment on two-phase (HCP and FCC) cobalt films with five different film thicknesses was investigated. Various tensile tests have shown that the deformation behavior of as-deposited cobalt films is different than for annealed films. After the heat treatment, the films exhibit a considerably higher onset strain for cracking reaching values between 4 and $7 \%$. For all films, the lattice strains increase with increasing engineering strain and decrease after $2 \%$ engineering strain. In the case of the asdeposited films, the lattice strains decrease due to crack formation as visible after reaching the crack onset strain of 1-2\%. In case of the annealed films, both, the strain-induced phase transformation with concomitant neck formation and, at higher engineering strains, also the formation of cracks causes the decrease of the lattice strains. Future experiments will be performed to quantify the contribution of both underlying mechanisms, necking or rather the phase transformation, which leads to the enhanced crack onset strains of the heat treated films. In all films independent of the heat treatment, the crack morphology through the film thickness is inclined by $45^{\circ}$ indicating shearing rather than brittle fracture and plastic deformation occurs in the as-deposited films. The deformation behavior changes in the annealed films from a ductile-like behavior for thicker films with a large amount of necking between the cracks to a more brittle-like behavior for the thinner films with channel cracking, absence of necking and pronounced buckling at higher strains. 
The heat treatment which stabilizes the FCC phase indeed favors plastic deformation in the form of necking and therefore a higher fracture strain. However, the crack formation increases dramatically at higher engineering strains compared to as-deposited cobalt films. Therefore, at application strains below $4 \%$, a heat treatment can significantly enhance the performance and reliability of the thin cobalt film systems.

\section{Acknowledgements}

The Helmholtz Zentrum für Materialien und Energie (Berlin, Germany) is acknowledged for partial funding (project number 2014_2_14201169). The authors thank B. Putz and O. Glushko (ESI, Leoben) for their help and support during the synchrotron measurements. G. Richter, F. Thiele and R. Völker (MPI for Intelligent Systems, Stuttgart, Germany) is acknowledged for fabricating the Co films with different film thicknesses. Additionally, the authors thank G. Bialkowski for annealing of the films and B. Breitbach for the phase analysis (MPIE, Düsseldorf, Germany).

\section{References}

[1] H. Chen, S.L. Whittenburg, Magnetic storage device using induced magnetic reversal of a cobalt element array, J. Appl. Phys. 94 (2003) 5278-5282.

[2] D.L. Ji, J.H. Li, L.M. Chen, D. Zhang, T. Liu, N. Zhang, R.Z. Ma, G.Z. Qiu, X.H. Liu, Needle-like CoO nanowires grown on carbon cloth for enhanced electrochemical properties in supercapacitors, RSC Advances 5 (2015) 41627-41630.

[3] M. Chen, X. Xia, J. Yin, Q. Chen, Construction of Co3O4 nanotubes as highperformance anode material for lithium ion batteries, Electrochim. Acta 160 (2015) $15-21$.

[4] S.B. Dalavi, R.N. Panda, Magnetic properties of Nanocrystalline Co and Ni synthesized via superhydride reduction route, J. Magn. Magn. Mater. 374 (2015) 411 416.

[5] M.A. Meyers, A. Mishra, D.J. Benson, Mechanical properties of nanocrystalline materials, Prog. Mater. Sci. 51 (2006) 427-556.

[6] A. Lisfi, J.C. Lodder, Microstructural and magnetic properties of metallic thin films obliquely sputtered on polymer, J. Magn. Magn. Mater. 242-245, Part 1 (2002) 370373.

[7] A.A. Karimpoor, U. Erb, K.T. Aust, G. Palumbo, High strength nanocrystalline cobalt with high tensile ductility, Scr. Mater. 49 (2003) 651-656. 
[8] A.A. Karimpoor, U. Erb, Mechanical properties of nanocrystalline cobalt, Physica Status Solidi (A) Applications and Materials Science 203 (2006) 1265-1270.

[9] P.A. Gruber, J. Böhm, F. Onuseit, A. Wanner, R. Spolenak, E. Arzt, Size effects on yield strength and strain hardening for ultra-thin $\mathrm{Cu}$ films with and without passivation: A study by synchrotron and bulge test techniques, Acta Mater. 56 (2008) 2318-2335.

[10] N. Lu, Z. Suo, J.J. Vlassak, The effect of film thickness on the failure strain of polymer-supported metal films, Acta Mater. 58 (2010) 1679-1687.

[11] D. Faurie, P.O. Renault, E. Le Bourhis, P. Goudeau, Study of texture effect on elastic properties of Au thin films by X-ray diffraction and in situ tensile testing, Acta Mater. 54 (2006) 4503-4513.

[12] I.M. Graz, D.P.J. Cotton, S.P. Lacour, Extended cyclic uniaxial loading of stretchable gold thin-films on elastomeric substrates, Appl. Phys. Lett. 94 (2009) 3435-3437.

[13] S. Olliges, S. Frank, P.A. Gruber, V. Auzelyte, H. Solak, R. Spolenak, Thermo mechanical properties and plastic deformation of gold nanolines and gold thin films, Mater. Sci. Eng., A 528 (2011) 6203-6209.

[14] M. Hommel, O. Kraft, Deformation behavior of thin copper films on deformable substrates, Acta Mater. 49 (2001) 3935-3947.

[15] P.A. Gruber, E. Arzt, R. Spolenak, Brittle-to-ductile transition in ultrathin Ta/Cu film systems, J. Mater. Res. 24 (2009) 1906-1918.

[16] M.J. Cordill, A. Taylor, J. Schalko, G. Dehm, Microstructure and adhesion of asdeposited and annealed Cu/Ti films on polyimide, Int. J. Mater. Res. 102 (2011) 729734.

[17] A.A. Taylor, M.J. Cordill, L. Bowles, J. Schalko, G. Dehm, An elevated temperature study of a Ti adhesion layer on polyimide, Thin Solid Films 531 (2013) 354-361.

[18] T. Tsuchiya, M. Hirata, N. Chiba, Young's modulus, fracture strain, and tensile strength of sputtered titanium thin films, Thin Solid Films 484 (2005) 245-250.

[19] W. Betteridge, The properties of metallic cobalt, Prog. Mater. Sci. 24 (1980) 51-142.

[20] A. Erko, I. Packe, C. Hellwig, M. Fieber-Erdmann, O. Pawlizki, M. Veldkamp, W. Gudat, KMC-2: the new x-ray beamline at BESSY II AIP Conf. Proc. 521 (2000) 415418.

[21] Helmholtz-Zentrum Berlin für Materialien und Energie, KMC-2: an X-ray beamline with dedicated diffraction and XAS endstations at BESSY II, Journal of large-scale research facilities 2 (2016) A49.

[22] H. Landolt, R. Börnstein, Numerical data and functional relationships in science and technology. Group III. Crystal and solid state physics: Springer, Berlin, 1979.

[23] P. Scherrer, Bestimmung der Größe und der inneren Struktur von Kolloidteilchen mittels Röntgenstrahlen, Nachrichten von der Gesellschaft der Wissenschaften zu Göttingen, Mathematisch-Physikalische Klasse / Zeitschriftenband (1918) 98-100.

[24] A.L. Patterson, The scherrer formula for X-ray particle size determination, Physical Review 56 (1939) 978-982.

[25] V.M. Marx, C. Kirchlechner, B. Breitbach, M.J. Cordill, D. Többens, T. Waitz, G. Dehm, Strain-induced phase transformation of a thin Co film on flexible substrates (2016) submitted.

[26] S.B. Lee, D.I. Kim, Y. Kim, S.J. Yoo, J.Y. Byun, H.N. Han, D.N. Lee, Effects of Film Stress and Geometry on Texture Evolution Before and After the Martensitic Transformation in a Nanocrystalline Co Thin Film, Metall. Mater. Trans. A 46 (2015) 1888-1899.

[27] G. Fleurier, E. Hug, M. Martinez, P.A. Dubos, C. Keller, Size effects and Hall-Petch relation in polycrystalline cobalt, Phil. Mag. Lett. 95 (2015) 122-130. 
[28] G.E. Ice, J.W.L. Pang, Tutorial on x-ray microLaue diffraction, Mater. Charact. 60 (2009) 1191-1201.

[29] V.M. Marx, F. Toth, A. Wiesinger, J. Berger, C. Kirchlechner, M.J. Cordill, F.D.

Fischer, F.G. Rammerstorfer, G. Dehm, The influence of a brittle Cr interlayer on the deformation behavior of thin $\mathrm{Cu}$ films on flexible substrates: Experiment and model, Acta Mater. 89 (2015) 278-289.

[30] J. Lohmiller, R. Baumbusch, O. Kraft, P.A. Gruber, Differentiation of Deformation Modes in Nanocrystalline Pd Films Inferred from Peak Asymmetry Evolution Using In Situ X-Ray Diffraction, Phys. Rev. Lett. 110 (2013) 0661011-0661015.

[31] S. Frank, U.A. Handge, S. Olliges, R. Spolenak, The relationship between thin film fragmentation and buckle formation: Synchrotron-based in situ studies and twodimensional stress analysis, Acta Mater. 57 (2009) 1442-1453.

Tables and Figures:

Table 1. Summary of the crystallite or rather grain sizes of the as-deposited and annealed cobalt films with different film thicknesses.

\begin{tabular}{lll}
\hline Sample & Film thickness $(\mathbf{n m})$ & Crystallite/Grain size (nm) \\
\hline As-deposited & 50 & $14 \pm 7 *$ \\
& 100 & $29 \pm 14 *$ \\
& 200 & $48 \pm 22^{*}$ \\
& 500 & $72 \pm 38 *$ \\
& 2000 & $91 \pm 32 *$ \\
Annealed & 50 & $126 \pm 42 * *$ \\
& 100 & $223 \pm 144 * *$ \\
& 200 & $276 \pm 147 * *$ \\
& 500 & $347 \pm 179 * *$ \\
& 2000 & $939 \pm 672 * *$ \\
\hline
\end{tabular}

* calculated by the X-ray peak width with the Scherrer equation (with $K=0.9$ ) from the synchrotron data

** determined from EBSD plane view measurements 

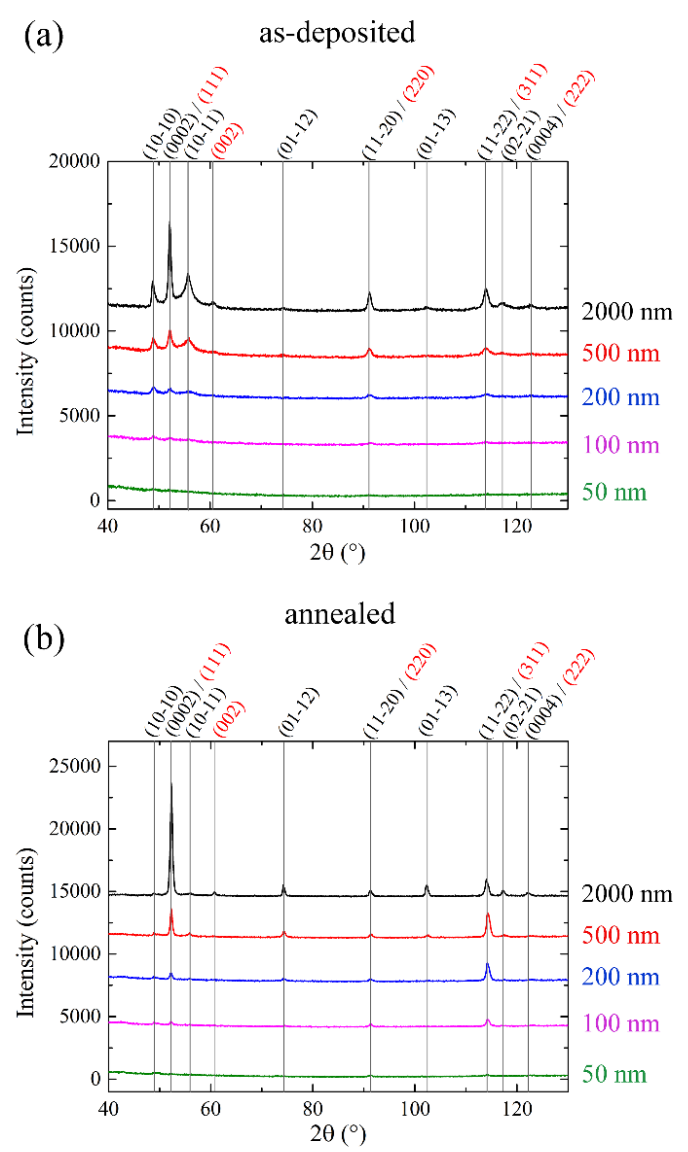

Figure 1. The $\theta-2 \theta$ curves of (a) the as-deposited and (b) the annealed films for all film thicknesses between 50 and $2000 \mathrm{~nm}$ measured under grazing incidence. The HCP peaks are marked in black, the FCC peaks in red.

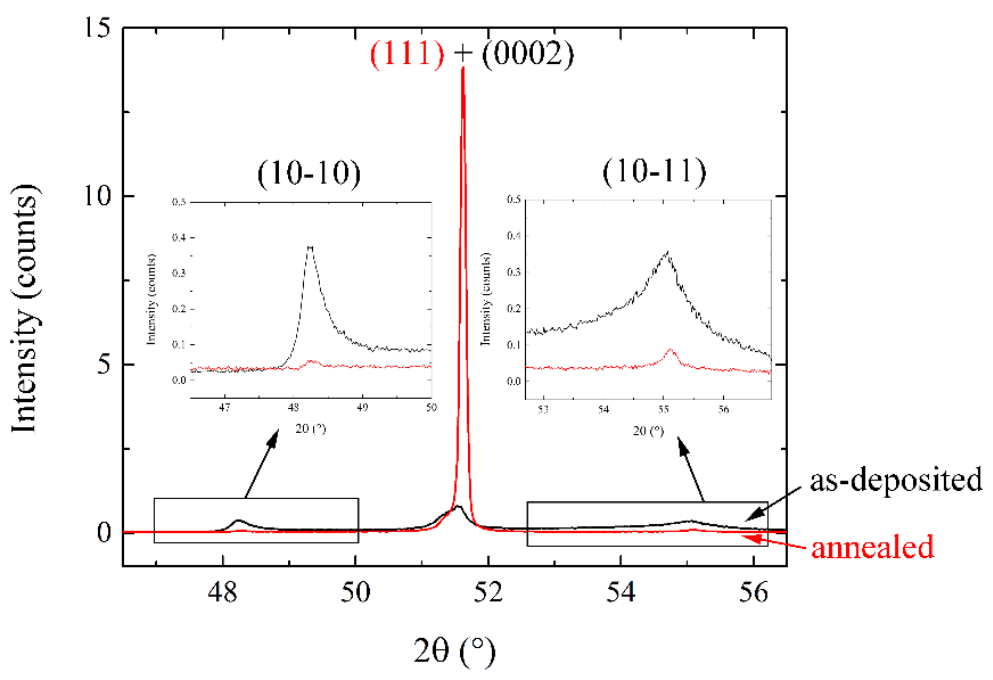

Figure 2. Zoom of the $2 \theta$ range between 46.5 and $56.5^{\circ}$. The $(10 \overline{1} 0)_{\mathrm{HCP}},(0002)_{\mathrm{HCP}} /(111)_{\mathrm{FCC}}$ and $(10 \overline{1} 1)_{\text {HCP }}$ peaks of the $2000 \mathrm{~nm}$ thick as-deposited (black) as well as annealed (red) cobalt film measured with synchrotron radiation. The inserts show an improved view of the $(10 \overline{1} 0)$ and $(10 \overline{1} 1)$ peak of the HCP phase. Note that $(111)_{\mathrm{FCC}}$ cobalt was observed at a Bragg angle of $51.25^{\circ}$ and $(0002)_{\mathrm{HCP}}$ at $51.6^{\circ}$, which cannot be discriminated. 


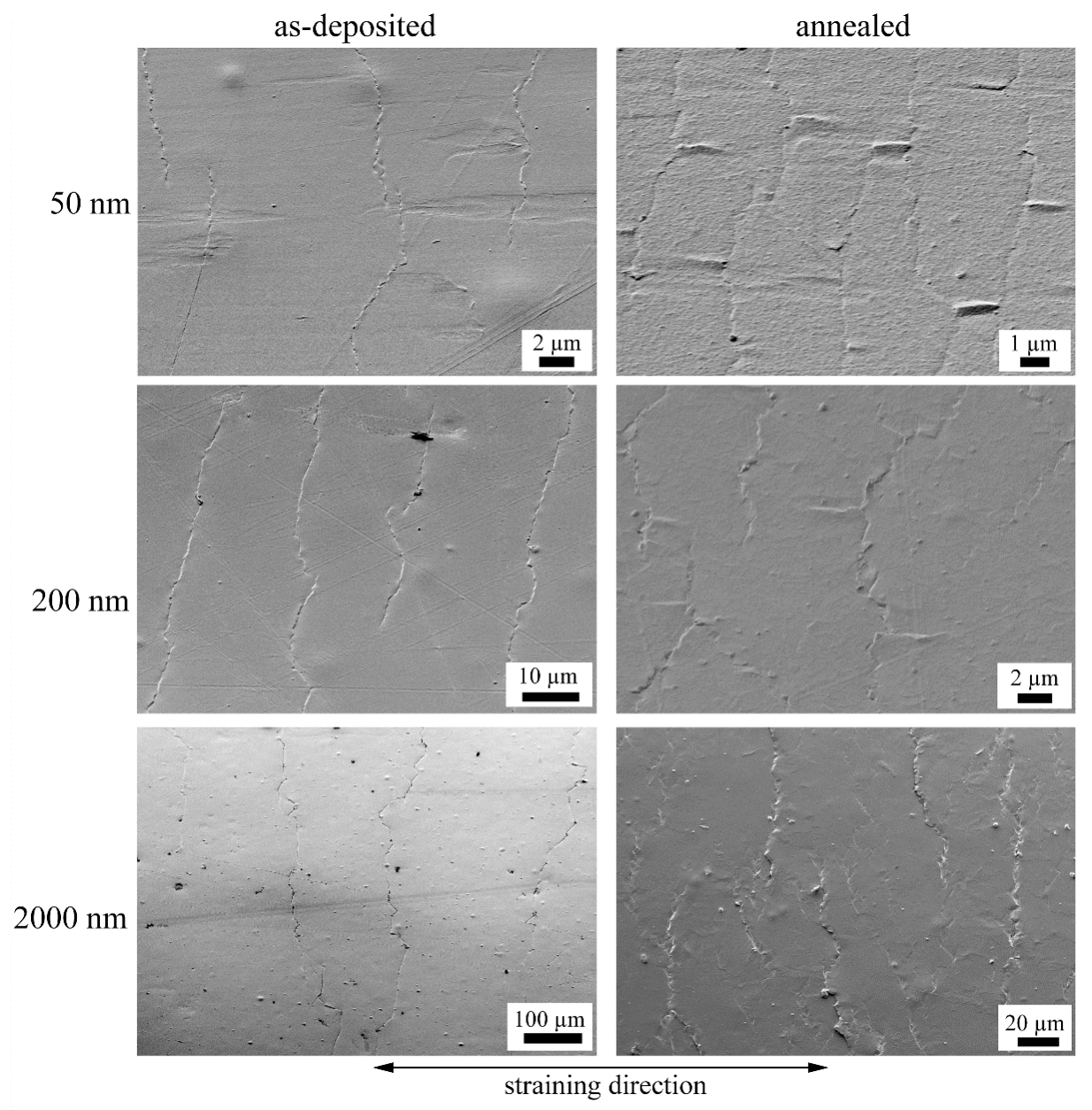

Figure 3. Post mortem SEM images of the 50, 200 and $2000 \mathrm{~nm}$ thick as-deposited and annealed films strained to $12 \%$ engineering strain recorded with the SE detector. Note that the scale bars are not equally-sized.

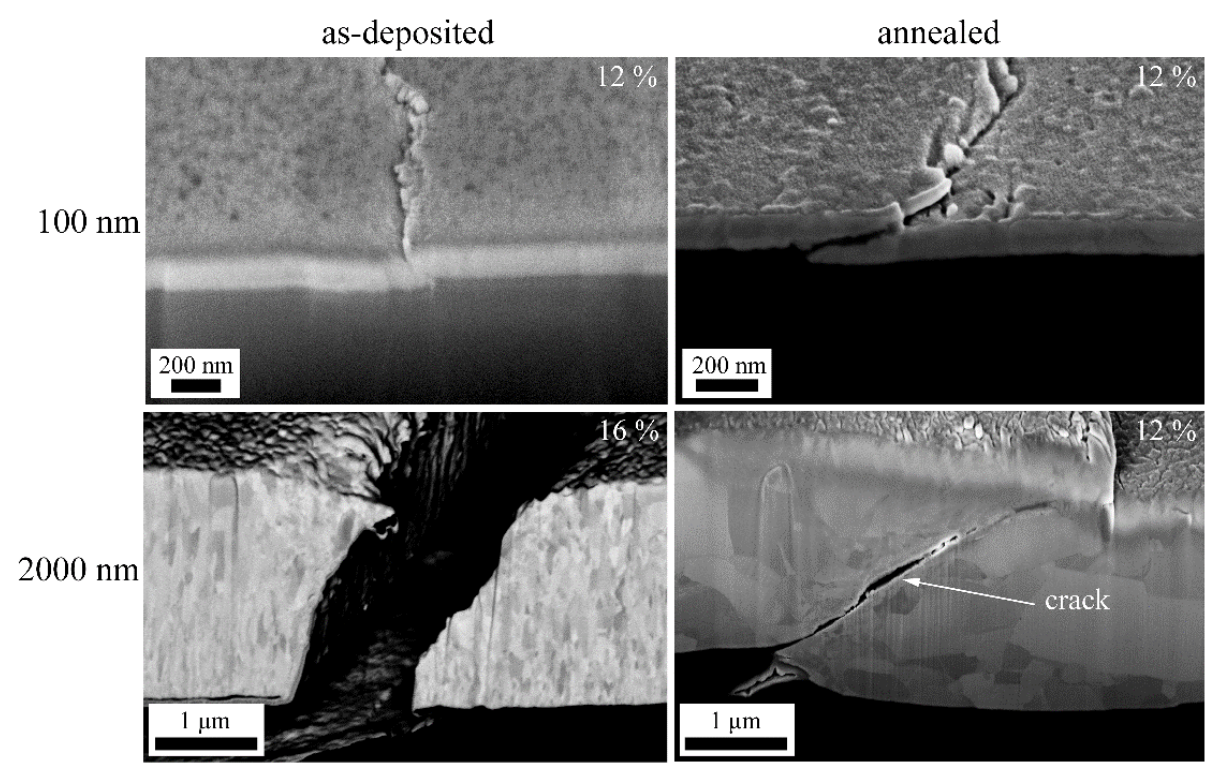

Figure 4. SEM images of cross sections through a crack of the 100 and 2000 nm thick asdeposited and annealed films captured with an Inlens detector. 


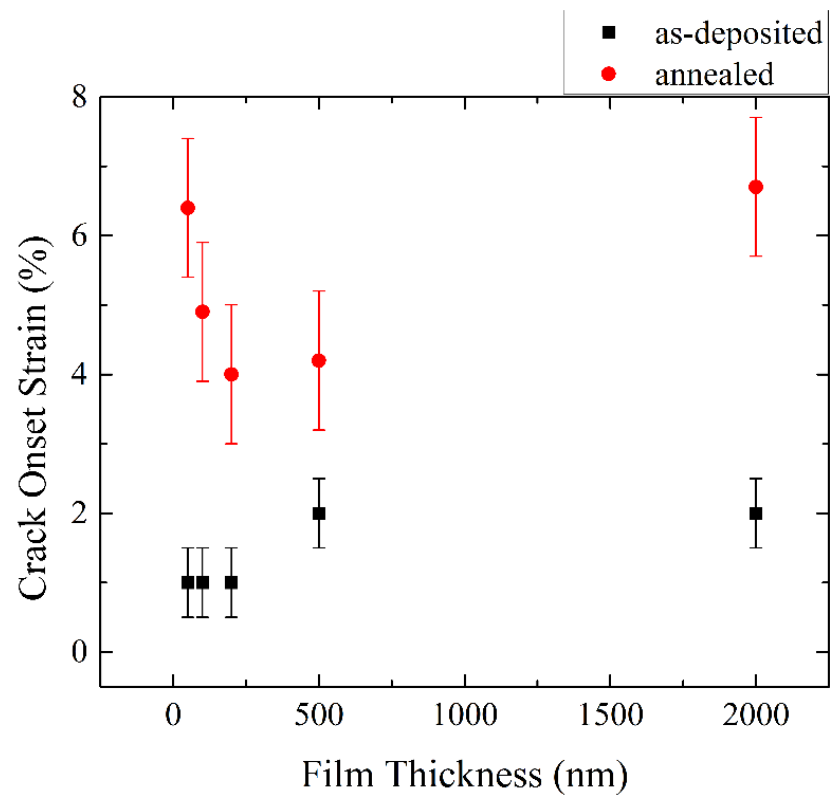

Figure 5. Crack onset strain of the as-deposited and annealed films determined by the in situ tensile tests under the optical light microscope. An error of $+/-0.5 \%$ for the as-deposited and $1 \%$ engineering strain for the annealed films is assumed.
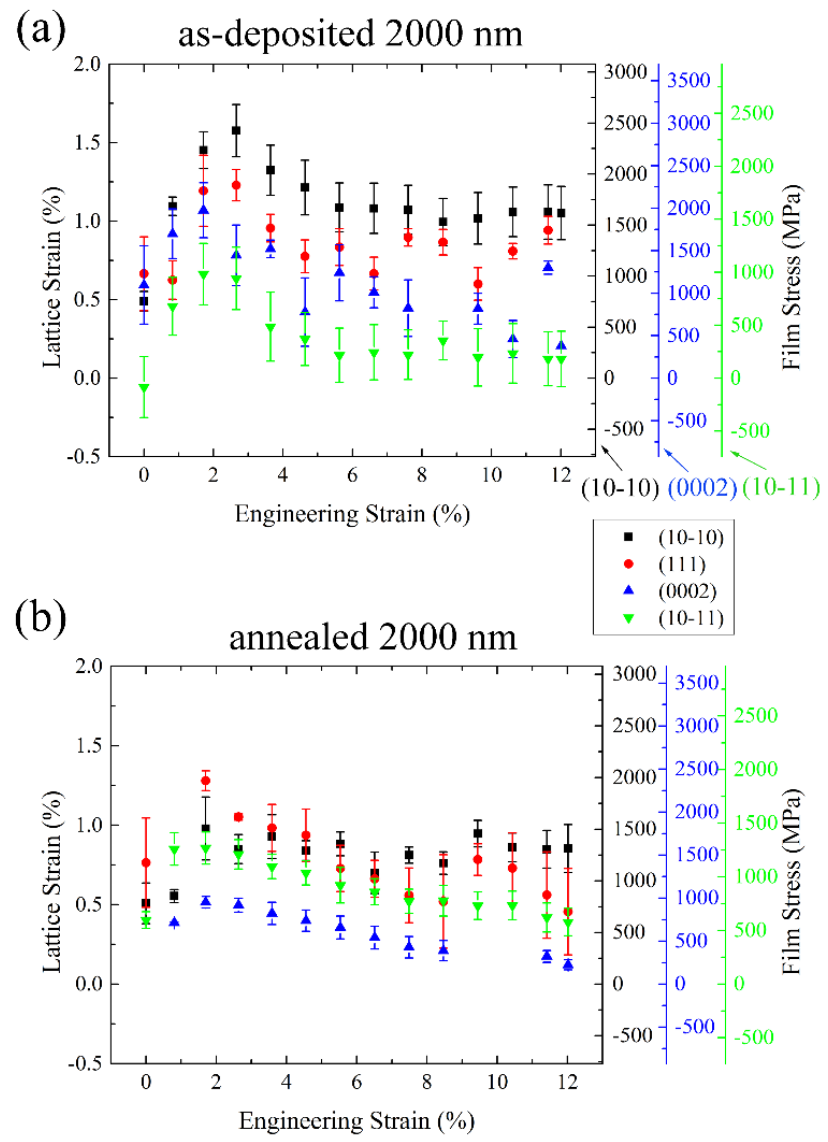

Figure 6. Lattice strains and film stresses calculated from the four different peaks shown in Figure 2 against the engineering strain of the (a) as-deposited and (b) annealed $2000 \mathrm{~nm}$ thick cobalt film. The film stresses are only determined for the HCP peaks. 

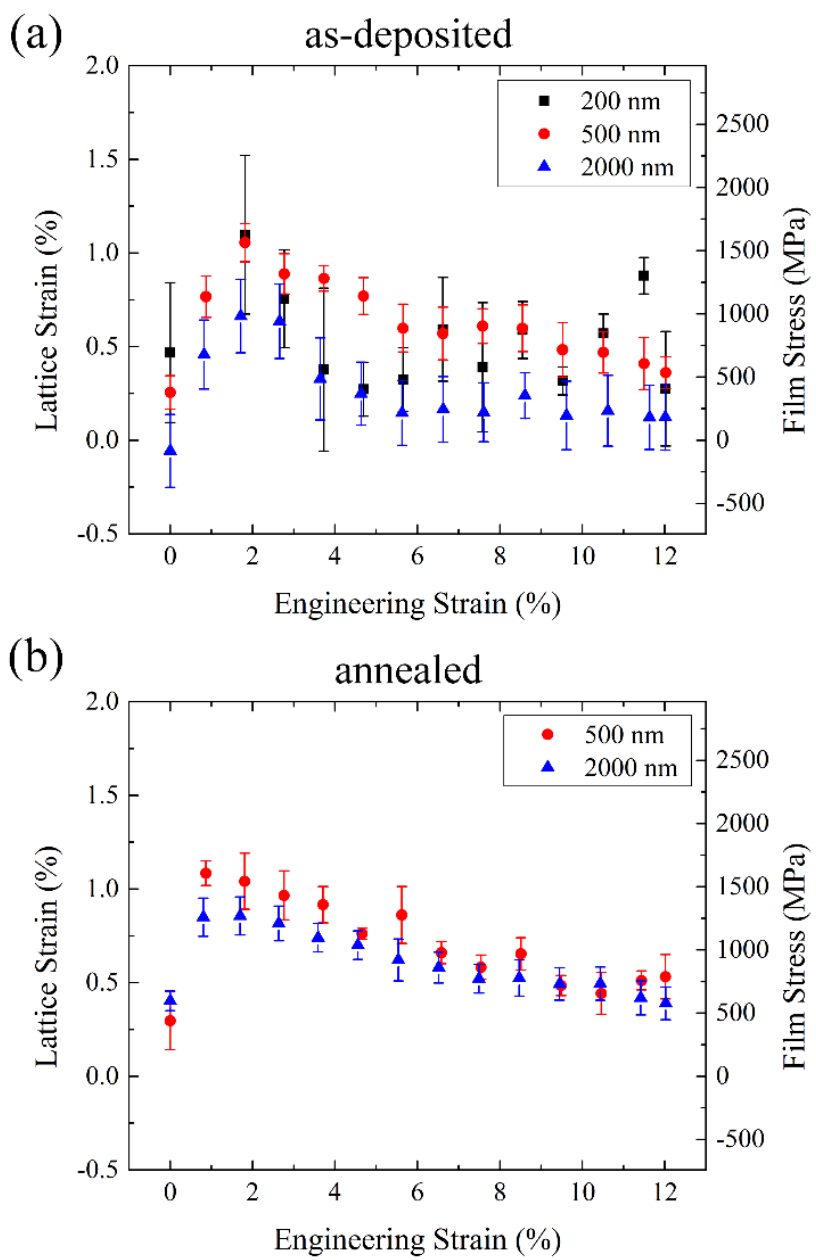

Figure 7. Lattice strains and film stresses calculated from the $(10 \overline{1} 1)_{\mathrm{HCP}}$ peak against the engineering strain of the (a) as-deposited and (b) annealed for different film thicknesses. 